\title{
Utility of whole-genome sequencing for detection of newborn screening disorders in a population cohort of 1,696 neonates
}

\author{
Dale L. Bodian, PhD ${ }^{1}$, Elisabeth Klein, DNP, RNC ${ }^{1}$, Ramaswamy K. lyer, $\mathrm{PhD}^{1,2}$, \\ Wendy S.W. Wong, PhD'1, Prachi Kothiyal, PhD ${ }^{1}$, Daniel Stauffer, PhD', \\ Kathi C. Huddleston, PhD, RN ${ }^{1}$, Amber D. Gaither, MD³ ${ }^{3}$ Irina Remsburg, MD², \\ Alina Khromykh, MD1, Robin L. Baker, MD4, George L. Maxwell, MD" ${ }^{5-7}$, \\ Joseph G. Vockley, PhD ${ }^{1,2}$, John E. Niederhuber, $\mathrm{MD}^{1,8}$ and Benjamin D. Solomon, $\mathrm{MD}^{1,3,9}$
}

Purpose: To assess the potential of whole-genome sequencing (WGS) to replicate and augment results from conventional blood-based newborn screening (NBS).

Methods: Research-generated WGS data from an ancestrally diverse cohort of 1,696 infants and both parents of each infant were analyzed for variants in 163 genes involved in disorders included or under discussion for inclusion in US NBS programs. WGS results were compared with results from state NBS and related follow-up testing.

Results: NBS genes are generally well covered by WGS. There is a median of one (range: 0-6) database-annotated pathogenic variant in the NBS genes per infant. Results of WGS and NBS in detecting
28 state-screened disorders and four hemoglobin traits were concordant for $88.6 \%$ of true positives $(n=35)$ and $98.9 \%$ of true negatives $(n=45,757)$. Of the five infants affected with a state-screened disorder, WGS identified two whereas NBS detected four. WGS yielded fewer false positives than NBS $(0.037$ vs. $0.17 \%)$ but more results of uncertain significance ( 0.90 vs. $0.013 \%$ ).

Conclusion: WGS may help rule in and rule out NBS disorders, pinpoint molecular diagnoses, and detect conditions not amenable to current NBS assays.

Genet Med advance online publication 3 September 2015

Key Words: genome; inborn errors of metabolism; newborn screening; next-generation sequencing; whole-genome sequencing

\section{INTRODUCTION}

Newborn screening (NBS) in the United States has been established on a state-by-state basis to efficiently and cost-effectively identify infants with conditions for which early treatment is available and necessary to ameliorate disease. Despite great success at identifying neonates at risk for the assessed conditions, NBS is not yet optimal. By design, NBS results in relatively high numbers of false positives in order to minimize false negatives. Additionally, "stressed" populations, such as sick and/or premature infants, can have higher false-positive rates, leading to increased NBS repetition and/or additional follow-up testing. ${ }^{1,2}$ Furthermore, the rarity, severity, and type of conditions included in NBS, as well as the purposeful skewing of NBS results toward inclusion rather than exclusion, lead to challenges in ensuring accurate diagnoses and can result in suboptimal medical care of infants and impose logistic, financial, and psychological burdens on families and health-care systems.
Improvements in next-generation sequencing (NGS) technologies, decreasing costs, and successes in diagnosing rare genetic disorders have raised the possibility of implementing NGS-whether whole-genome, exome, or genepanel sequencing - as part of NBS programs. Currently, blood-based NBS is performed using methods that measure metabolites, enzyme activity, or other molecules. DNA sequencing is limited to second-tier testing and/or the subsequent diagnostic workup, and may not be included in the recommended diagnostic algorithm. ${ }^{3}$ Cited advantages of incorporating NGS into NBS include (i) expanding the set of mutations assessed by second-tier NBS DNA sequencing, which is often performed on a limited number of genes and their most common mutations, (ii) the ability to add tests for other disorders efficiently and at minimal additional cost, including those that are not readily amenable to current assays, (iii) the availability of the newborn's genomic

\footnotetext{
${ }^{1}$ Inova Translational Medicine Institute, Inova Health System, Falls Church, Virginia, USA; ${ }^{2}$ Department of Obstetrics and Gynecology, Virginia Commonwealth University School of Medicine, Richmond, Virginia, USA; ${ }^{3}$ Department of Pediatrics, Inova Children's Hospital, Inova Health System, Falls Church, Virginia, USA; ${ }^{4}$ Fairfax Neonatal Associates, Inova Health System, Falls Church, Virginia, USA; ${ }^{5}$ Department of Obstetrics and Gynecology, Inova Fairfax Hospital, Falls Church, Virginia, USA; ${ }^{6}$ Women's Health Integrated Research Center at Inova Health System, Falls Church, Virginia, USA; ${ }^{7}$ Department of Defense Gynecologic Cancer Center of Excellence, Annandale, Virginia, USA; ${ }^{8}$ Johns Hopkins University School of Medicine, Baltimore, Maryland, USA; ${ }^{9}$ Department of Pediatrics, Virginia Commonwealth University School of Medicine, Richmond, Virginia, USA. Correspondence: John E. Niederhuber (john.niederhuber@inova.org) or Dale L. Bodian (dale.bodian@inova.org)
} 
Table 1 Cohort demographics and genomic data

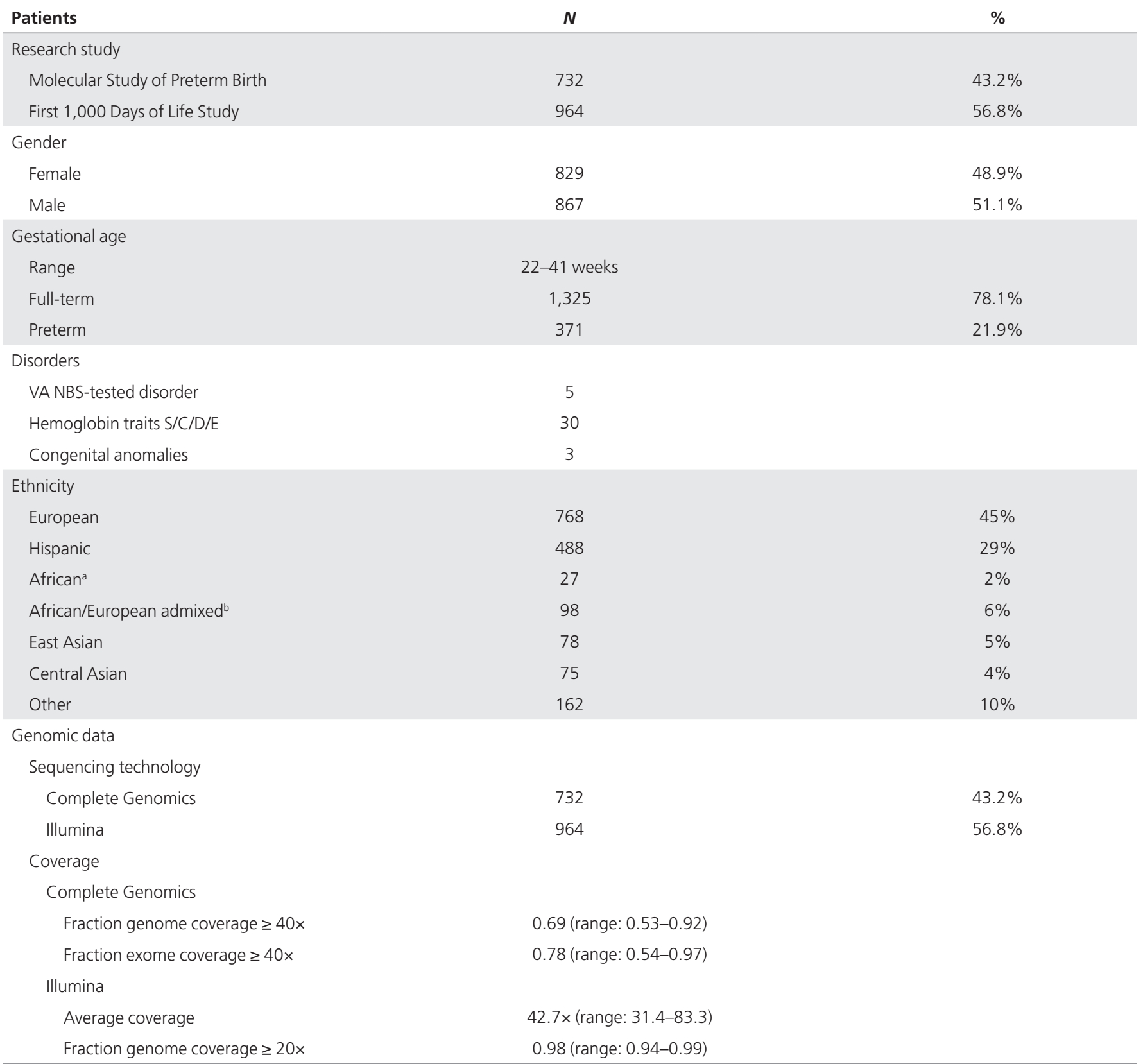

NBS, newborn screening; VA, Commonwealth of Virginia.

andividuals with $>78 \%$ African ancestry by genomic admixture analysis. IIndividuals with $13-75 \%$ African ancestry and $21-87 \%$ European ancestry by genomic admixture analysis.

information throughout life, and (iv) reducing the total number of tests performed. ${ }^{4,5}$

Despite the perceived benefits, genomic sequencing of newborns raises a number of ethical, regulatory, legal, economic, and technical issues, and the decision of whether to implement NGS as part of an NBS program requires assessment of all these factors. Some of these issues have been discussed elsewhere, such as in recent publications on ethics ${ }^{6}$ and $\operatorname{cost}^{7}$ of NGS for NBS. With recent proof-of-principle demonstrations that data can be generated within 1-3 days, the major challenge for clinical application of NGS now centers on analysis and interpretation. ${ }^{5,8,9}$ Technical limitations affecting coverage and variant detection also impact comprehensive and accurate detection of disease-causing variants. ${ }^{5}$ Limited data are currently available on how these analytical issues affect the performance of NGS for population-based NBS.

To help address the question of the utility of incorporating NGS more primarily into NBS, we present a comparison of the results of whole-genome sequencing (WGS) analysis with results from conventional NBS. We performed the analysis on 
an ethnically and racially diverse population cohort of 1,696 neonates with (i) whole-genome sequences from the infants and both parents, (ii) state NBS results, and (iii) electronic health record (EHR) and other relevant medical-history data, including clinical diagnoses and results of follow-up newborn testing. The cohort includes affected, preterm, and, importantly, healthy infants. Our analysis of the analytic performance of WGS for NBS-related disorders, emphasizing detection of disease-causing variants and clinical interpretation, provides much needed information useful for deciding whether, when, and how to implement NGS in NBS programs.

\section{Study participants and clinical data}

\section{MATERIALS AND METHODS}

Family trios composed of a neonate and both parents were recruited at Inova Fairfax Hospital in Virginia during 2011-2014 and enrolled in either the Molecular Study of Preterm Birth or the First 1,000 Days of Life Study. Both studies were conducted by the Inova Translational Medicine Institute and approved by the Western Institutional Review Board (studies 1124761 and 20120204). Informed consent was obtained for all study participants for research uses of their medical and genomic data, including review of their medical record. A combined cohort was assembled for this NBS study composed of neonates with available state NBS results and research-generated WGS data from both parents (Table 1). Individuals were unselected for NBS-related disorders. Biological parentage was confirmed genomically, and ancestry was computed from the genomic data as described. ${ }^{10}$

State NBS results, confirmatory tests, and clinical diagnoses of NBS disorders were manually extracted from the neonates' EHR. Data not available from the EHR were obtained from the pediatrician of record when possible and from longitudinal health surveys from the First 1,000 Days of Life Study. Results of all NBS tests, including repeats, were recorded, as were results of tests that were done to follow-up or otherwise rule-in or ruleout conditions based on NBS findings. Results from NBS tests indicating transfusion of blood products within 8 weeks prior to sample collection were classified as invalid and included in the counts of uncertain results. Data supporting the diagnoses of affected neonates are summarized in Supplementary Table S1 online.

\section{Sample processing and WGS}

Peripheral blood was collected from each family member and DNA extracted as described. ${ }^{10}$ WGS, assembly, and variant calling for the Molecular Study of Preterm Birth samples were performed by Complete Genomics (CG) (Mountain View, CA) using the Complete Genomics' Assembly Pipeline versions 2.0.0-2.0.3 with the NCBI build 37 human reference assembly. ${ }^{11}$ The 1,000 Days of Life Study samples were sequenced by Illumina (San Diego, CA) with the Illumina Whole Human Genome Sequencing Service Informatics Pipeline version 2.0102 using the Isaac package (https://github.com/sequencing) ${ }^{12}$ and the hg19 human reference genome. ${ }^{11}$ Single-nucleotide polymorphisms, substitutions, and indels called by the vendors were normalized ${ }^{13}$ with GATK $^{14}$ version 2.8.1 (https://www. broadinstitute.org/gatk/), then quality-filtered. Genotypes were required to be fully called and to have a read depth $\geq 10$, allele balance $\geq 0.25$, and quality scores GQ $\geq 30$ for Illumina and equal allele fraction score $\geq 46$ for $C G$.

\section{List of NBS genes}

Conditions were first selected based on all conditions included in current NBS in the United States or in development, as annotated by the American College of Medical Genetics and Genomics. ${ }^{3}$ Selections of the genes and relevant mode(s) of inheritance corresponding to these conditions were based on manual review of conditions and corresponding genes listed in the Clinical Genomic Database, ${ }^{15,16}$ Online Mendelian Inheritance in Man (OMIM) ${ }^{17}$ and Genetics Home Reference. ${ }^{18}$ Additional genes from work by Bhattacharjee et al., ${ }^{4}$ were also included. Genes and conditions pertinent to the state where participating infants were born were cross-referenced with state department of health resources. ${ }^{19}$ The list of genes and conditions is provided in Supplementary Table $\mathbf{S 2}$ online, with the genes associated with disorders screened by Virginia indicated.

\section{Annotation of variant pathogenicity}

Variants in the NBS genes were extracted using coordinates on the hg19 reference assembly for the NBS genes retrieved from the UCSC KnownGenes table, ${ }^{20}$ extended by 1,000 bases into promoter regions. Ambiguity in the genomic mapping of CYP21A2 due to the presence of the highly homologous pseudogene CYP21A1P were resolved according to the Entrez Gene mapping. ${ }^{21}$

Variants were annotated as pathogenic, likely pathogenic, of uncertain significance, likely benign, or benign using data from four sources: (i) ClinVar, a curated database that links genomic variants to clinical phenotypes that includes benign as well as pathogenic variants and aggregates data from OMIM, locus-specific databases, and genetic testing laboratories; ${ }^{22}$ (ii) G6PD-MutDB; ${ }^{23}$ (iii) predicted protein impact; and (iv) predicted deleteriousness of nonsynonymous single-nucleotide variants.

For ClinVar annotations, clinical significance was obtained from the XML file, version 2014-11. Variants with both pathogenic and benign annotations for the same disorder were mapped to uncertain significance, as were variants with clinical significance not provided. Metadata and provided GRCh37 genomic coordinates and alleles for single-nucleotide variants, small insertions and deletions, and indels were extracted where available. Variants present in ClinVar solely because of previous submissions from our cohort were excluded. Missing or inconsistent alleles were converted to the hg19 reference sequence using SAMtools ${ }^{24}$ v1.1 (https://github.com/samtools/samtools). After splitting multiallelic loci, variants were left-shifted with GATK $^{14}$ and normalized. Haplotype designations were retained. Inheritance patterns and disease names were extracted and mapped to a controlled vocabulary (Supplementary Table S2 
online). Inheritance values missing from ClinVar were retrieved as described above.

Because of legacy data in ClinVar misassigning pathogenicity to individual variants comprising well-characterized haplotypes in $G 6 P D$, pathogenicity annotations for variants in this gene were obtained from G6PD-MutDB. ${ }^{23}$ Similarly, the variants comprising the Duarte variant of GALT were assigned "pathogenic" as a haplotype but benign individually. For simplicity, we refer to the variants with pathogenicity annotations from ClinVar or G6PD-MutDB as "database-annotated."

Variants were also annotated with ANNOVAR $^{25}$ version 2014-07-14 (http://annovar.openbioinformatics.org) using the refGene database, which includes all annotated RefSeq ${ }^{26}$ transcripts. Predicted nonsense, consensus splice site, and frameshift variants that were not database-annotated received preliminary annotations of likely pathogenic. Likely pathogenic or uncertain significance annotations for all variants were updated to likely benign if the minor allele frequency in the cohort was $>1 \%$. The ClinVar variants affected are listed in Supplementary Table $\mathbf{S 3}$ online.
The fourth annotation source was deleteriousness as predicted by $\mathrm{LR}^{27}$ using ANNOVAR. ${ }^{25}$ The deleteriousness annotations were used for statistics only and were not considered in predicting affected status for the NBS disorders based on the genomic data.

\section{Genomic prediction of affected status}

Affected status was assigned to each neonate for each disorder using an entirely automated method based on the annotated pathogenicity of the filtered variants, the inheritance of the disorder, and the phase of the variants computed from the parental genomes. For recessive disorders, infants with two pathogenic or likely pathogenic high-quality variants in the same gene associated with the same condition were predicted to be affected if the two variants were unambiguously determined to be in trans. If phase could not be determined, the infants were classified as "possibly affected" for the disorder. For dominant disorders, affected status was assigned given a single pathogenic or likely pathogenic variant in a relevant gene. X-linked recessive disorders were treated as dominant

a

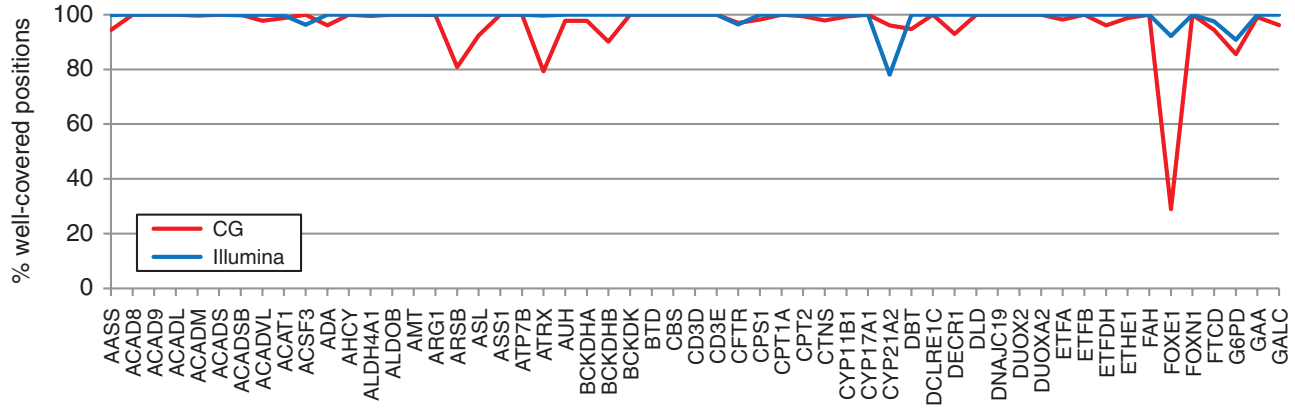

b

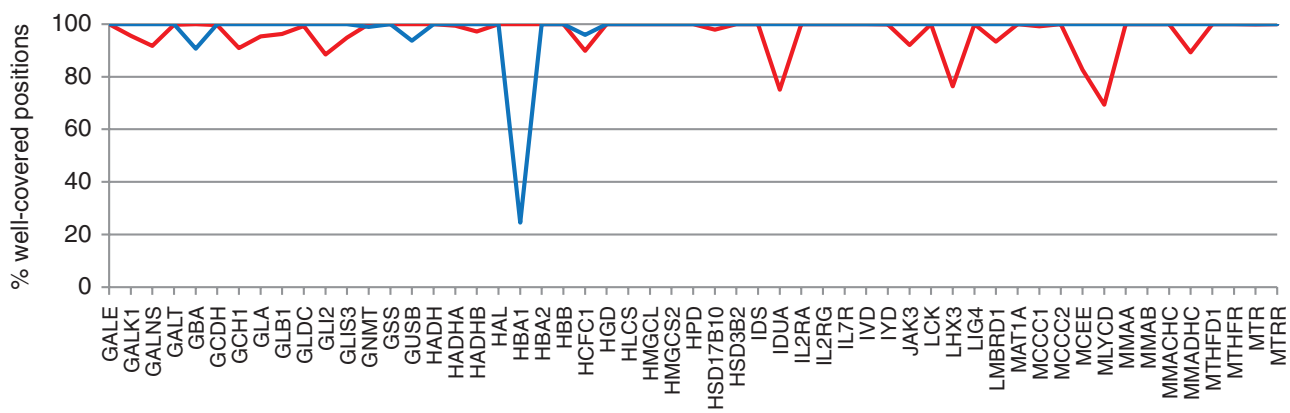

C

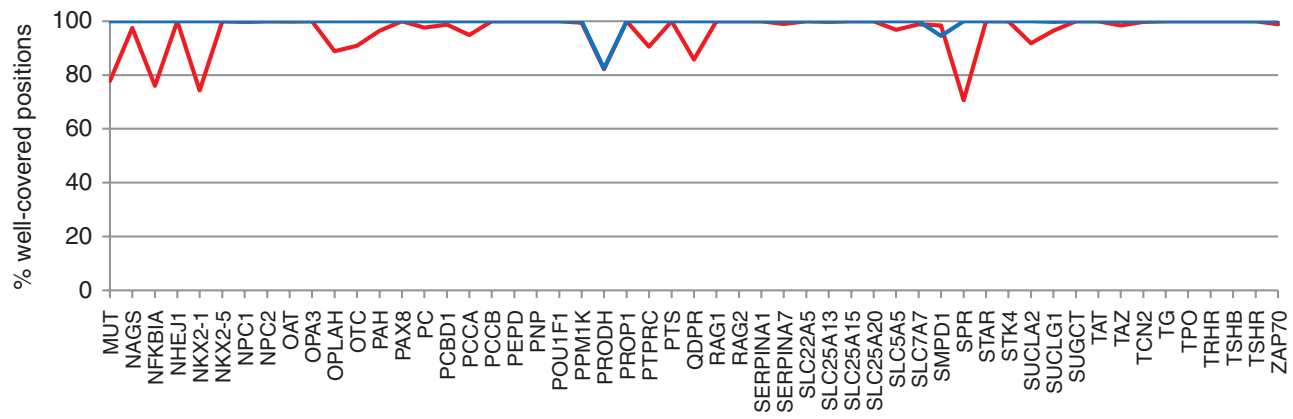

Figure 1 Coverage of NBS genes. Percentage of exonic positions in each gene that are well-covered in $>95 \%$ of the genomes. Genes are ordered alphabetically by gene symbol. (a) AASS-GALC; (b) GALE-MTRR; (c) MUT-ZAP70. Red, CG genomes; blue, lllumina genomes. CG, Complete Genomics; NBS, newborn screening. 
for males and recessive for females. For all disease states, haplotypes were treated as one variant. Infants with variants of uncertain significance (VUS) who would be considered affected if the VUS were pathogenic were assigned a status of uncertain for the relevant disorders.

\section{Coverage calculations}

Coverage statistics for the CG genomes were calculated using the weight-sum sequence coverage depth. For the Illumina genomes, coverage was determined using the GATK ${ }^{14}$ version 2.8.1 CallableLoci command with parameters $\operatorname{minDepth}=10$, minBaseQuality=5, and minMappingQuality=3. Coverage was calculated for every exonic position in any transcript associated with an NBS gene in the UCSC Genome Browser KnownGenes table $^{20}$ except for CYP21A2, which was limited to the Entrez Gene mappings. ${ }^{21}$ A "well-covered" genomic position was defined as having $\geq 10$ passing reads in $>95 \%$ of the genomes sequenced.

\section{Variant call concordance}

Concordance calculations are described in the Supplementary Methods online.

\section{RESULTS}

\section{Participants and genomic data}

To evaluate the performance of WGS for NBS in a population cohort, we analyzed high-quality whole-genome sequences from newborns enrolled in our research studies for whom results of state NBS and related follow-up testing were available. A total of 1,696 newborn subjects were available for analysis after exclusion of 15 sequenced infants lacking complete NBS and/or full follow-up test results. The cohort is predominantly healthy and ethnically and racially diverse, and includes both full-term and preterm infants (Table 1). Genomes were sequenced to $>60 \times$ and $>40 \times$ coverage by CG and Illumina, respectively (Table 1 ). Based on validation studies of the parental genomes, we estimated the variant false-positive rate to be $<1.3 \%$ for false negatives and

a

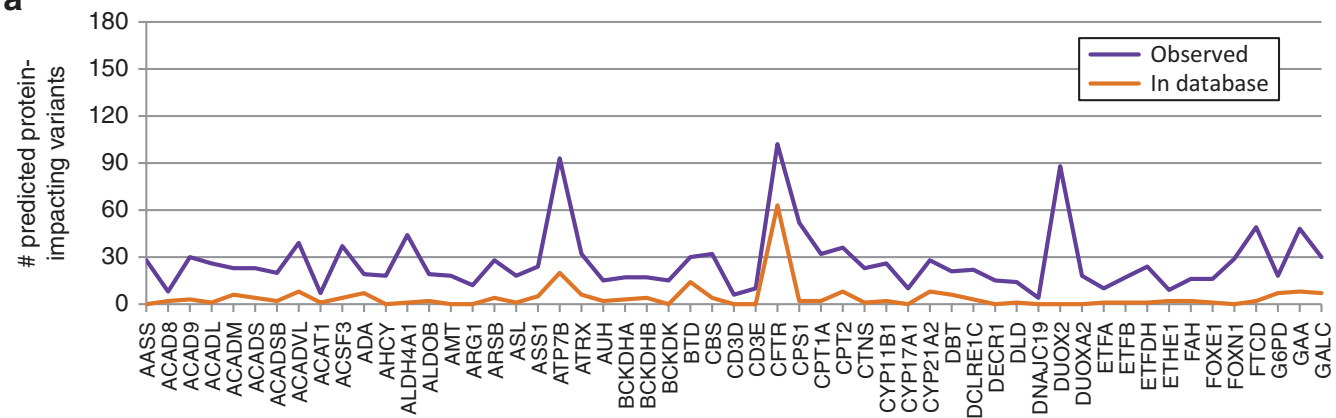

b

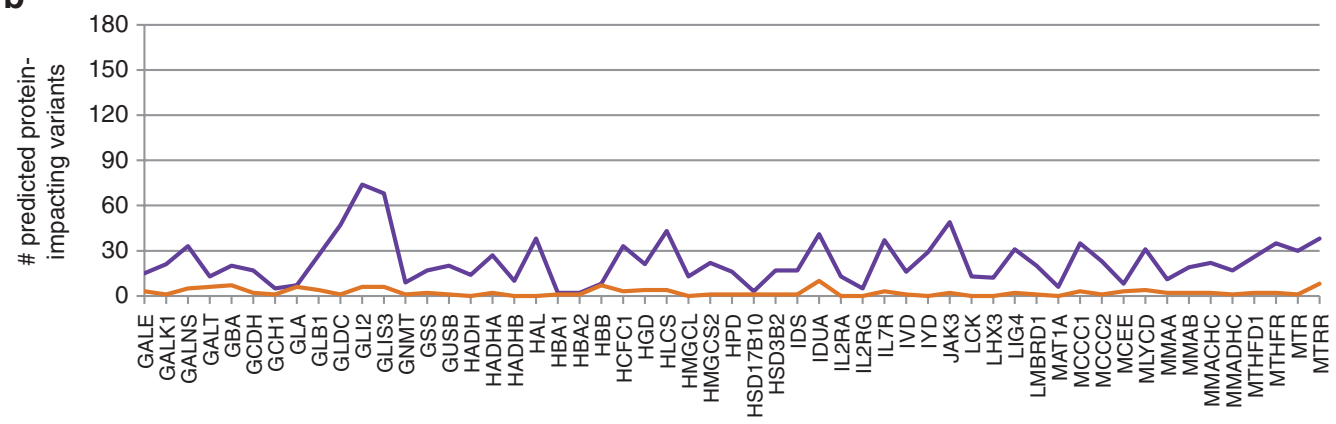

C

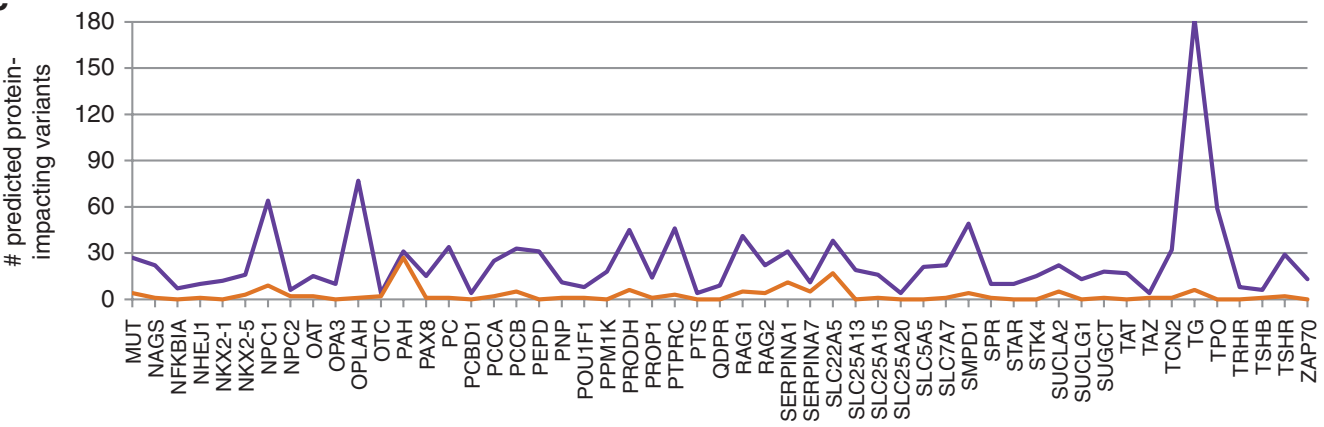

Figure 2 Representation of observed variants in clinical phenotype database. Genes are ordered alphabetically by gene symbol. (a) AASS-GALC; (b) GALE-MTRR; (c) MUT-ZAP70. Purple, number of distinct predicted protein-affecting variants per gene observed; gold, number of observed predicted protein-affecting variants that are represented in ClinVar or G6PD-MutDB. 
$<2.3 \%$ for false positives for the CG data. ${ }^{10}$ The discordance rate of variant calls in the NBS genes in 66 infants sequenced by both CG and Illumina is 0.00277 , 95\% confidence interval (0.00258, 0.00295 ) for single-nucleotide polymorphisms and $0.0134,95 \%$ confidence interval $(0.0123,0.0146)$ for indels (Supplementary Figure S1 online) (the Illumina genomes for these 66 infants were used only for the concordance-rate calculation, not the other analyses presented).

\section{WGS coverage of NBS genes}

The exonic coverage of the 163 NBS genes varied by gene and sequencing technology (Figure 1). In the CG data, 80\% (130) of the NBS genes met the minimum coverage level in $>95 \%$ of the genomes at $>95 \%$ of their coding positions, and $51 \%$ (83) were completely well covered. FOXE1 is the only gene with fewer than half $(29 \%)$ of its exonic bases well covered by CG. For the Illumina genomes, 95\% (155) of the genes had $>95 \%$ well-covered exonic positions, and 88\% (144) were completely well covered. The single gene with fewer than half its exonic bases well covered by Illumina is HBA1 (25\%). Coverage within genes varied by exon (Supplementary Figure $\mathbf{S 2}$ online), consistent with observations from other gene sets. ${ }^{28}$

However, disease-associated variants are not distributed evenly throughout genes. Of 2,650 exonic pathogenic database-annotated variants in the NBS genes, 2,575 (97\%) were well covered by CG and 2,636 (99\%) by Illumina. Pathogenic variants well covered in fewer than half of the genomes were in genes $G B A$ (exon 11) and HBA1 (exon 2) by Illumina and CFTR (exon 15) and MYLCD (exon 1) by CG.

\section{Variants in NBS genes}

We observed 260,029 distinct variants in the 163 NBS genes, of which 4,021 are predicted to impact the protein sequence. The number of predicted protein-impacting variants in the cohort varies by gene (Figure 2), from 182 in $T G$ and 102 in CFTR to 1 in each of HBA1 and HBA2. The infants carry a median of 93 (range: 69-123) predicted protein-impacting variants and a median of 1 (range: $0-6$ ) database-annotated pathogenic variant in the set of 163 genes. No reported pathogenic variants in the NBS genes were detected in 750 (44\%) of the neonates.
Because interpretation of the clinical impact of sequence variants is highly dependent on database annotations, we calculated the number of predicted protein-impacting NBS-gene variants observed in the studied genomes that are represented in ClinVar or G6PD-MutDB. A median of 54\% of the variants per infant (range: $38-66 \%$ ) are captured in these databases. Counted by gene, the median percentage of database-represented variants is $7.7 \%$ (range: $0-88 \%$ ) (Figure 2), of which 79\% (394 of the 500 observed database-annotated variants) have pathogenicity annotations that are not identified as uncertain. Among the genes with greater than five predicted protein-impacting variants in the cohort, $H B B, P A H, G L A$, and CFTR have the most complete database representation but are still incomplete, with $13-38 \%$ of the observed variants not yet included.

\section{Concordance of WGS and NBS results}

Next, we compared the results from the genomic analysis with the final results of blood-based NBS achieved after completion of indicated repeat screening, as well as with clinical diagnoses extracted from the EHR. For this comparison, the genomic analysis was limited to the subset of 65 NBS genes related to the disorders screened by the Commonwealth of Virginia (VA) blood-based program (Supplementary Table S2 online). The results of the comparison are summarized in Table 2, and the variants, NBS results, and clinical diagnoses are presented in Supplementary Table S4 online. All candidate disease-associated variants in affected patients were orthogonally confirmed, as were $18 / 19$ variants that led to a false-positive genomic prediction in patients with an available sample and $2 / 2$ representative variants associated with hemoglobin traits (Supplementary Methods, Table S4, and Table S5 online).To quantitate the results, we multiplied 27 disorders (the 26 non-hemoglobin disorders screened by VA, plus one more representing both the hemoglobinopathies screened and hemoglobin traits S, C, D, and E) by 1,696 infants for a total of 45,792 results. There is generally good agreement between WGS and NBS results, with $88.6 \%$ of the true positives $(n=35)$ and $98.9 \%$ of the true negatives $(n=45,757)$ called correctly by both. There were 513 results for which the results of the two methods disagreed, of which $80 \%$ (409) were genomic uncertain results derived from variants of unknown pathogenicity;

Table 2 Comparison of the NBS results and genomic analysis

NBS

\begin{tabular}{|c|c|c|c|c|c|c|}
\hline & & \\
\hline & & True positive & False negative & Uncertain & False positive & True negative \\
\hline \multirow{3}{*}{ Genome sequencing } & False negative & $2(0.004 \%)$ & $0(0 \%)$ & $0(0 \%)$ & $0(0 \%)$ & NA \\
\hline & False positive & NA & $0(0 \%)$ & $0(0 \%)$ & $0(0 \%)$ & $17(0.037 \%)$ \\
\hline & True negative & NA & NA & $6(0.013 \%)$ & $77(0.168 \%)$ & $45248(98.81 \%)$ \\
\hline
\end{tabular}

NBS-reportable beta-hemoglobinopathies and hemoglobin traits were counted as one disorder. NBS results are defined as the final results of the screening tests and do not include results of second-tier tests. Compound heterozygous Duarte variant galactosemia, but not heterozygous Duarte variant carriers, were scored as reportable by NBS.

NA, not applicable; BS, newborn screening. 
a partially overlapping $16 \%$ (81) were NBS false positives, 3\% (17) were genomic false positives, and one was due to a miscalled indel in PCCB. The two methods were complementary in their uncertain calls, with no disorders given uncertain results by both.

Of the five individuals known to be affected by a condition (although not necessarily a medically actionable condition) expected to be captured by VA NBS, WGS identified two (40\%), including one case of suspected Duarte variant galactosemia missed by conventional NBS and one infant with hemoglobin SC disease. WGS also correctly identified all 30 individuals with hemoglobin traits S, C, D, or E detected by conventional NBS.

Three positive cases were missed by WGS. For the infant reported to have long-chain 3-hydroxyacyl-CoA dehydrogenase deficiency/trifunctional protein deficiency based on conventional NBS, genomic analysis identified a single mutation in the HADHB gene, c.992delT (NM_001281513). The identical heterozygous mutation was identified by clinical sequencing of $H A D H A$ and $H A D H B$ by GeneDx (Gaithersburg, MD) that was performed as part of clinical genetic follow-up. Neither the clinical sequencing nor targeted CGH array identified a second mutation, and the patient continues to lack a satisfying molecular diagnosis. All exons in $H A D H A$ and $H A D H B$ were well covered in this infant's genome. The second affected patient missed by WGS was clinically diagnosed with congenital hypothyroidism. The genomics identified a single de novo VUS in NKX2-1, c.596C>G (NM_001079668) p.S199W, leading to an uncertain result. The same result was obtained by clinical trio-based exome sequencing and was interpreted as likely pathogenic in the context of medical issues identified later, including neurologic anomalies. No other predicted protein-impacting variant in a relevant gene was found. The third affected patient was clinically diagnosed with salt-wasting congenital adrenal hyperplasia. No predicted protein-impacting variant was found in any of the genes associated with this disorder, possibly owing to difficulties sequencing the pseudogene-containing genomic region of CYP21A2, the gene involved in most cases of congenital adrenal hyperplasia. ${ }^{29}$

WGS gave false-positive predictions of affected status for 17 neonates, 15 of which resulted from a database-annotated (2) or predicted likely pathogenic (13) variant in a gene for a dominant disorder (Supplementary Table S4 online). Thirteen of these cases are due to variants in DUOX2 and two to NKX2-5. The remaining two false-positive predictions resulted from a pair of database-annotated pathogenic variants in trans in a single gene, one pair in BTD and the other in CFTR. Review of the BTD variants revealed that one, c. $880 \mathrm{~A}>\mathrm{G}$, is currently labeled as uncertain in the submitting locus-specific database, ${ }^{30}$ consistent with a false-positive genomic result for this infant. The two CFTR variants, c. $1666 \mathrm{~A}>\mathrm{G}$ (p.I556V) and c.4056G $>\mathrm{C}$ (p.Q1352H), are molecularly deleterious, but their clinical relevance to cystic fibrosis is unclear. ${ }^{31}$ None of the 17 neonates had positive NBS results for these conditions, and signs of these conditions were ruled out at $>1$ year of age via review of the EHR and study-specific surveys. In comparison, the conventional
NBS tests had 81 false positives, $80 \%$ (65) of which were from two markers, T4 and IRT.

One of the concerns regarding implementation of NGS for NBS is the potential for high numbers of results of uncertain significance. Conventional NBS gave $0.013 \%$ (6) uncertain results, compared with $0.90 \%$ (410) for WGS, which would impact $23.6 \%$ of the infants. Most $(88 \%, 359)$ of the genomic uncertain calls resulted from a VUS in a gene linked to a dominant disorder, with $83 \%$ (298) of these from two genes, DUOX2 and GLI2, both of which can result in hypothyroidism, and the latter of which can also involve additional phenotypic findings. ${ }^{32}$ Both genes have poor database representation, with $0 \%(0 / 88)$ and $8 \%(6 / 74)$ of the observed variants, respectively, included. Of the 261 distinct variants leading to uncertain results, $24.1 \%$ are predicted to be deleterious by an ensemble prediction method. Limited to recessive disorders, $2.9 \%$ (50) of the infants received an uncertain result. The 51 pairs of VUS are from 14 genes (Supplementary Table S4 online).

The availability of the parental genome sequences aided the prediction of affected status for recessive disorders by enabling calculation of the phase of pathogenic variants in the child. Three potentially positive WGS results were excluded by phasing with the parental genomes. The sequences of the parents are also useful for identifying false positives that may result from sequencing error. Of the 13 pathogenic or likely pathogenic variants that led to false-positive results, two variants were not present in either parent; both were insertions in DUOX2.

\section{Number of collections and gestational age}

The 1,696 infants in the cohort underwent 2,075 NBS screens and 190 second-tier tests, an average of 1.3 sample collections (range: 1-24) per neonate. The number of collections was strongly dependent on term $(P<2 \mathrm{e}-16$ by $t$-test), with preterm infants undergoing twice as many collections on average as full-term neonates ( 1.08 vs. 2.26 ; median: 1 vs. 2 ). The term-dependence was not limited to a single test but was apparent for all VA NBS markers with abnormal results from at least four impacted individuals (Figure 3c). To compare this result with that of WGS, we calculated the number of sample collections by counting all WGS runs, including resequencing due to quality issues (although these did not require additional collections of research samples), plus one collection for a hypothesized follow-up test for each disorder with an abnormal or uncertain result from the genomic analysis. By this method, WGS had an average of 1.3 collections per infant (range: $1-3$ ), with the number of collections independent of term (mean full-term: 1.28, mean preterm: $1.32, P=0.11$ by $t$-test; Figure $3 \mathbf{b}$ ).

\section{DISCUSSION}

The decision regarding whether, how, and when to implement NGS as part of NBS is complex, requiring consideration of many factors, including efficacy, efficiency, cost, and bioethical concerns, and would benefit from data-derived evidence evaluating each aspect. Our assessment of the analytic performance of WGS for current and planned NBS conditions compared 


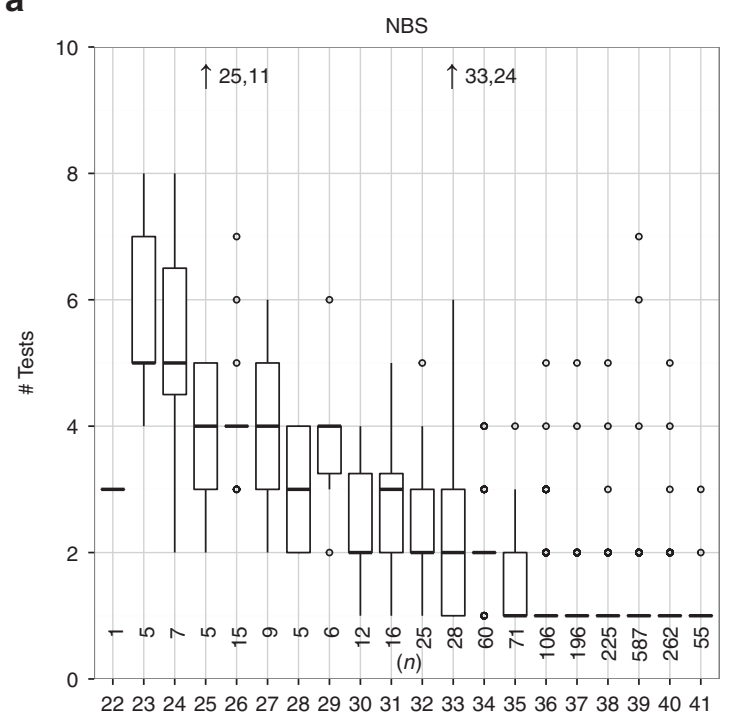

b

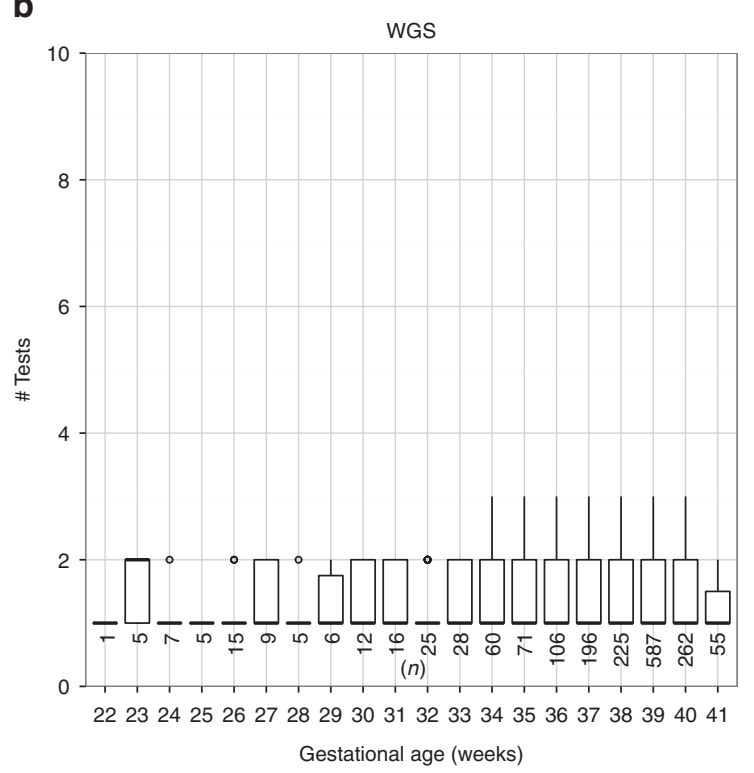

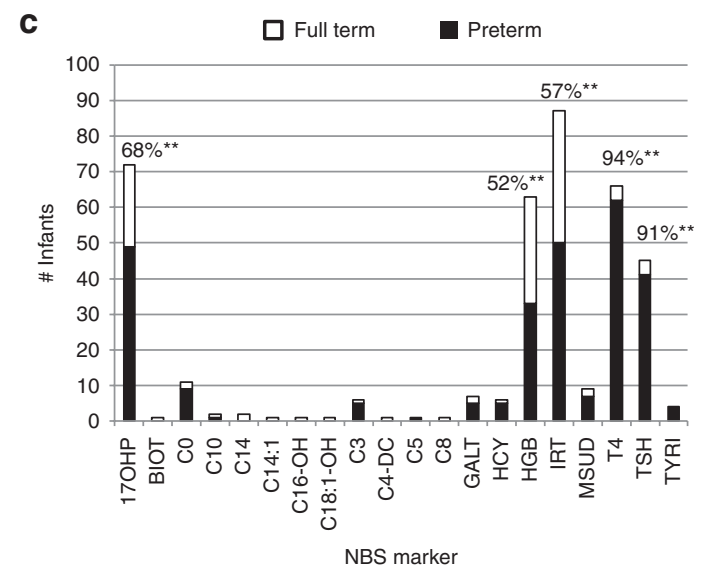

Figure 3 Effect of gestational age on NBS and WGS. Number of (a) NBS tests, including repeat screens and second-tier tests, and (b) WGS samples per neonate. WGS counts include lab failures plus one hypothesized test representing a confirmatory test for each VA NBS gene with a predicted result of affected, possibly affected, or unknown. Off-scale values in a are indicated by an arrow labeled with gestational age, number of tests. Dark bars represent the median, and the boxes span the first and third quartiles. $n=$ number of infants in each gestational age group. (c) Number of infants with at least one abnormal or invalid result for each VA NBS tested marker. Filled bars, preterm; open bars, full-term, \%, percentage of preterm infants. **Statistically significant enrichment of preterm individuals $(P<=1$ e- 5 by chi-squared test), limited to tests with $n \geq 4$ for all cells. NBS, newborn screening; VA, Commonwealth of Virginia; WGS, whole-genome sequencing.

with conventional NBS demonstrates the strengths and complementarity of these technologies.

In this study, conventional NBS identified more affected neonates than WGS did ( $4 / 5$ vs. $2 / 5)$. However, the difference is not statistically significant due to the low number of patients affected with these rare disorders in our population cohort. This success rate of our completely automated WGS analysis pipeline for NBS disorders is comparable to that reported for WES-based diagnosis of patients with suspected genetic disorders, which uses protocols dependent on variant prioritization by experts and congruity with clinical manifestations-a feature not appropriate for NBS, which aims for diagnosis before the onset of clinical signs. ${ }^{33}$

WGS was shown to be complementary to conventional NBS because (i) it gave fewer false-positive results, (ii) it resolved the inconclusive results from conventional NBS, (iii) it identified the causative mutations in affected individuals and was able to distinguish disorders indistinguishable by conventional NBS, and (iv) fewer sample collections were required to achieve the results for preterm infants.

As seen here, WGS can provide precise molecular diagnoses for Mendelian disorders with clear genetic causes. Although conventional NBS may not be able to provide definitive underlying causes for certain conditions, it is able to identify individuals with conditions that are nongenetic in origin. For example, congenital hypothyroidism may be related to genetic causes that result in isolated congenital hypothyroidism, syndromic disorders that involve congenital hypothyroidism with other findings affecting multiple organ systems, and nongenetic causes. 
Another significant advantage of WGS over conventional NBS is that it can identify individuals affected by or at risk for a larger number of disorders than are encompassed by current NBS. For example, we identified 14 infants with genomically predicted glucose-6-phosphate dehydrogenase deficiency, a condition that is not included in our state's NBS program. Diagnosing Mendelian disease through broad genetic testing may directly benefit patient care, allow more informed medical decisions, and aid family planning. ${ }^{34}$ Availability of WGS data might also eventually be helpful for the infants for considerations outside of NBS. Although technically feasible, genome-wide testing of infants for all known genetic disorders remains controversial for ethical, legal, and social reasons. ${ }^{5-7}$ Other NGS methods with more limited scope, such as targeted gene panels, are also being evaluated and may mitigate some of these concerns.

The performance of NGS, whether whole-genome or targeted, will continue to improve as the platforms and software mature. Efforts are under way to improve coverage; variant calling, including copy number and other structural variants; phasing; and interpretation, including broadening our understanding of disease pathogenesis..$^{29,35-37}$ Periodic reassessment of the performance of NGS for NBS would be appropriate. These evaluations would benefit from testing a much larger number of affected neonates than were available in this cohort, in addition to healthy and preterm infants of diverse ancestry as was done here. These reassessments could also monitor the cost of NGS, including sequencing, interpretation, and infrastructure expenses, which are currently prohibitive for large-scale adoption but are expected to continue to decrease. ${ }^{5}$

NGS is a disruptive technology that will have an impact on $\mathrm{NBS}^{38}$ and will affect the practice of medicine in general. Sequencing is already being performed with increasing frequency as part of follow-up testing for abnormal NBS results and in other clinical and research-based efforts. Studies such as the one reported here are critical for ensuring that the implementation of this technology into routine clinical care proceeds efficiently and effectively, so as to maximize benefit to patients and health-care systems.

\section{SUPPLEMENTARY MATERIAL}

Supplementary material is linked to the online version of the paper at http://www.nature.com/gim

\section{ACKNOWLEDGMENTS}

This work was funded by the Inova Health System, with support from Fairfax County and generous philanthropic support from the Odeen family. We thank the physicians and staff of Inova Cares Clinic for Women, Karen R. Maser MD, LLC, Kathy Wolf, MD, Nova Group for Women, Perinatal Associates of Northern Virginia, and Rajiv Baveja for their support of the studies. We also thank Susan Flynn, Radhika Hastak, and Alex Ashchenko for help with clinical data extraction; Rishi Haridas for Sanger sequencing; Inova Translational Medicine Institute staff for study support; and
Larry Brody and Tiina Urv for comments on the manuscript. The authors and contributors express their gratitude to all patients and families taking part in the Inova Translational Medicine Institute's genomic studies.

\section{DISCLOSURE}

The authors declare no conflict of interest.

\section{REFERENCES}

1. Slaughter JL, Meinzen-Derr J, Rose SR, et al. The effects of gestational age and birth weight on false-positive newborn-screening rates. Pediatrics 2010;126:910-916.

2. Tarini BA, Clark SJ, Pilli S, et al. False-positive newborn screening result and future health care use in a state Medicaid cohort. Pediatrics 2011;128:715-722.

3. American College of Medical Genetics. ACMG ACT Sheets and Confirmatory Algorithms. American College of Medical Genetics: Bethesda, MD, 2001.

4. Bhattacharjee A, Sokolsky T, Wyman SK, et al. Development of DNA confirmatory and high-risk diagnostic testing for newborns using targeted nextgeneration DNA sequencing. Genet Med 2015;17:337-347.

5. Landau YE, Lichter-Konecki U, Levy HL. Genomics in newborn screening. J Pediatr 2014;164:14-19.

6. Knoppers BM, Sénécal K, Borry $P$, Avard D. Whole-genome sequencing in newborn screening programs. Sci Trans/ Med 2014;6:229cm2.

7. Beckmann JS. Can we afford to sequence every newborn baby's genome? Hum Mutat 2015;36:283-286.

8. Saunders CJ, Miller NA, Soden SE, et al. Rapid whole-genome sequencing for genetic disease diagnosis in neonatal intensive care units. Sci Trans/ Med 2012;4:154ra135.

9. Stranneheim H, Engvall M, Naess K, et al. Rapid pulsed whole genome sequencing for comprehensive acute diagnostics of inborn errors of metabolism. BMC Genomics 2014;15:1090.

10. Bodian DL, McCutcheon JN, Kothiyal $P$, et al. Germline variation in cancersusceptibility genes in a healthy, ancestrally diverse cohort: implications for individual genome sequencing. PLOS ONE 2014;9:e94554.

11. Lander ES, Linton LM, Birren B, et al.; International Human Genome Sequencing Consortium. Initial sequencing and analysis of the human genome. Nature 2001;409:860-921.

12. Raczy C, Petrovski R, Saunders $C T$, et al. Isaac: ultra-fast whole-genome secondary analysis on Illumina sequencing platforms. Bioinformatics 2013:29:2041-2043.

13. Tan A, Abecasis GR, Kang HM. Unified representation of genetic variants Bioinformatics 2015;31:2202-2204.

14. McKenna A, Hanna M, Banks E, et al. The Genome Analysis Toolkit: a MapReduce framework for analyzing next-generation DNA sequencing data. Genome Res 2010;20:1297-1303.

15. Solomon BD, Nguyen AD, Bear KA, Wolfsberg TG. Clinical genomic database. Proc Natl Acad Sci USA 2013:110:9851-9855.

16. National Human Genome Research Institute. Clinical Genomic Database. http:// research.nhgri.nih.gov/CGD/. Accessed 14 February 2015.

17. Online Mendelian Inheritance in Man, OMIM®. McKusick-Nathans Institute of Genetic Medicine, Johns Hopkins University: Baltimore, MD. http://omim.org/. Accessed 14 February 2015

18. National Institutes of Health. Genetics Home Reference. http://ghr.n/m.nih.gov/ Accessed 14 February 2015.

19. Virginia Department of Health. Newborn Screening. http://www.vdh.virginia gov/ofhs/childandfamily/childhealth/gns/vnsp/. Accessed 15 November, 2014.

20. Karolchik D, Barber GP, Casper J, et al. The UCSC Genome Browser database: 2014 update. Nucleic Acids Res 2014;42(Database issue):D764-D770.

21. National Center for Biotechnology Information. Gene.http://www.ncbi.nlm.nih. gov/gene. Accessed 26 February, 2015.

22. Landrum MJ, Lee JM, Riley GR, et al. ClinVar: public archive of relationships among sequence variation and human phenotype. Nucleic Acids Res 2014:42(Database issue):D980-D985.

23. Zhao X, Li Z, Zhang X. G6PD-MutDB: a mutation and phenotype database of glucose-6-phosphate (G6PD) deficiency. J Bioinform Comput Bio/ 2010;8(suppl 1):101-109.

24. Li H, Handsaker B, Wysoker A, et al.; 1000 Genome Project Data Processing Subgroup. The Sequence Alignment/Map format and SAMtools. Bioinformatics 2009:25:2078-2079. 


\section{ORIGINAL RESEARCH ARTICLE}

25. Wang K, Li M, Hakonarson H. ANNOVAR: functional annotation of genetic variants from high-throughput sequencing data. Nucleic Acids Res 2010;38:e164.

26. Pruitt KD, Brown GR, Hiatt SM, et al. RefSeq: an update on mammalian reference sequences. Nucleic Acids Res 2014;42(Database issue):D756-D763.

27. Dong C, Wei P, Jian X, et al. Comparison and integration of deleteriousness prediction methods for nonsynonymous SNVs in whole exome sequencing studies. Hum Mol Genet 2015:24:2125-2137.

28. Park JY, Clark P, Londin E, Sponziello M, Kricka LJ, Fortina P. Clinical exome performance for reporting secondary genetic findings. Clin Chem 2015;61: 213-220.

29. Mueller PW, Lyons J, Kerr G, Haase CP, Isett RB. Standard enrichment methods for targeted next-generation sequencing in high-repeat genomic regions. Genet Med 2013;15:910-911.

30. University of Utah Department of Pathology. Biotinidase Deficiency and BTD. http://arup.utah.edu/database/BTD/BTD_welcome.php. Accessed 31 March 2015.

31. Lee JH, Choi JH, Namkung W, et al. A haplotype-based molecular analysis of CFTR mutations associated with respiratory and pancreatic diseases. Hum $\mathrm{Mol}$ Genet 2003;12:2321-2332.

32. Bear KA, Solomon BD, Antonini S, et al. Pathogenic mutations in GLI2 cause a specific phenotype that is distinct from holoprosencephaly. J Med Genet 2014:51:413-418.

33. Yang Y, Muzny DM, Xia F, et al. Molecular findings among patients referred for clinical whole-exome sequencing. JAMA 2014;312:1870-1879.

34. Kingsmore SF, Dinwiddie DL, Miller NA, Soden SE, Saunders CJ. Adopting orphans: comprehensive genetic testing of Mendelian diseases of childhood by next-generation sequencing. Expert Rev Mol Diagn 2011;11:855-868.
35. Boycott KM, Vanstone MR, Bulman DE, MacKenzie AE. Rare-disease genetics in the era of next-generation sequencing: discovery to translation. Nat Rev Genet 2013;14:681-691.

36. Marshall E. Biomedicine. NIH seeks better database for genetic diagnosis. Science 2013;342:27

37. Rimmer A, Phan H, Mathieson I, et al.; WGS500 Consortium. Integrating mapping-, assembly- and haplotype-based approaches for calling variants in clinical sequencing applications. Nat Genet 2014:46:912-918.

38. Bailey DB Jr, Gehtland L. Newborn screening: evolving challenges in an era of rapid discovery. JAMA 2015:313:1511-1512.

(1) $@ \Theta$ This work is licensed under a Creative Commons Attribution-NonCommercial-NoDerivs $\quad 4.0$ International License. The images or other third party material in this article are included in the article's Creative Commons license, unless indicated otherwise in the credit line; if the material is not included under the Creative Commons license, users will need to obtain permission from the license holder to reproduce the material. To view a copy of this license, visit http://creativecommons.org/licenses/by-nc-nd/4.0/ 